\title{
Modifications in graphene electron states due to a deposited lattice of Au nanoparticles: Density functional calculations
}

\author{
Sabrina S. Carara, ${ }^{1}$ Ronaldo J. C. Batista, ${ }^{2}$ and Hélio Chacham ${ }^{1, *}$ \\ ${ }^{1}$ Departamento de Física, ICEX, Universidade Federal de Minas Gerais, CP 702, 30123-970 Belo Horizonte, MG, Brazil \\ ${ }^{2}$ Departamento de Física, Universidade Federal de Ouro Preto, Campus Morro do Cruzeiro, 35400-000 Ouro Preto, MG, Brazil
}

(Received 28 March 2009; revised manuscript received 18 August 2009; published 29 September 2009)

\begin{abstract}
We perform first-principles investigations of two-dimensional, triangular lattices of $\mathrm{Au}_{38}$ nanoparticles deposited on a graphene layer. We find that lattices of thiolate-covered nanoparticles cause electronic structure modifications in graphene such as minigaps, charge transfer, and new Dirac points, but graphene remains metallic. In contrast, for a moderate coverage of nanoparticles $\left(\approx 0.2 \mathrm{~nm}^{-2}\right)$, a lattice of bare (noncovered) Au nanoparticles may induce periodic deformations on the graphene layer leading to the opening of a band gap of a few tens of $\mathrm{meV}$ at the Dirac point, in such a way that a properly charged system might become a semiconductor.
\end{abstract}

DOI: 10.1103/PhysRevB.80.115435

PACS number(s): 73.22.-f, 61.46.Df, 73.90.+f

\section{INTRODUCTION}

The electronic properties of graphene-based materials have been widely investigated since graphene was first synthesized in 2004. ${ }^{1}$ Due to graphene's unique dispersion relation, electrons and holes behave like a two-dimensional (2D) gas of massless Dirac fermions, which allows to probe quantum electrodynamics phenomena in condensed matter. ${ }^{2-4} \mathrm{On}$ the other hand, the metallic (or zero-gap semiconductor) nature of graphene's electronic structure prevents its direct application as a "bulk" semiconductor material in electronic devices. Several experimental realizations have been made to modify graphene and turn it into a semiconductor, such as cutting or synthesizing graphene in the form of nanoribbons, ${ }^{5}$ growing graphene epitaxially on $\mathrm{SiC},{ }^{6,7}$ or applying an electric field perpendicular to bilayer graphene. ${ }^{8}$ It has also been suggested that graphene with periodic arrays of antidots ${ }^{9}$ or $\mathrm{BN}$-graphene bilayers ${ }^{10}$ might manifest semiconducting behaviors.

In the present work we investigate, through firstprinciples calculations, the consequences of depositing a two-dimensional triangular lattice of $\mathrm{Au}$ nanoparticles on graphene. Such triangular lattices of functionalized nanoparticles can be produced experimentally, ${ }^{11}$ and the deposition of Au nanoparticles on graphene has already been obtained. ${ }^{12}$ We find that deposited $\mathrm{Au}_{38}$ nanoparticles that are covered with methylthiolate molecules transfer a net negative charge to graphene and cause electronic structure modifications such as new Dirac-type points and minigaps, but graphene remains metallic. In the case of lattices of bare (noncovered) Au nanoparticles, the stronger nanoparticle-graphene interaction may lead to the opening of a band gap of a few tens of $\mathrm{meV}$ at the graphene Dirac point for moderate concentrations of nanoparticles.

\section{METHODOLOGY}

We use a first-principles methodology based on the density functional theory ${ }^{13}$ as implemented in the SIESTA code, ${ }^{14}$ within the generalized gradient approximation (GGA) (Ref. $15)$ and with the use of norm-conserving pseudopotentials. ${ }^{16}$
For the $\mathrm{C}, \mathrm{S}$, and $\mathrm{H}$ atoms we employ a basis set consisting of double-zeta radial functions per angular momentum, with the finite range determined by a common confinement energy shift in $\delta E=10^{-2}$ Ry (Ref. 17). For Au atoms, we use a different basis set, which is described in previous works. ${ }^{18,19}$ The real-space grid used for integration was defined by a minimal energy cutoff of 150 Ry (Ref. 20) in the reciprocal space.

\section{RESULTS AND DISCUSSION}

\section{A. Deposited layers of functionalized nanoparticles: Band structure near the Fermi level}

We first consider two-dimensional arrays of functionalized $\mathrm{Au}_{38}$ nanoparticles deposited on graphene, as shown in Fig. 1. We consider the nanoparticle structure obtained experimentally by Garzon et al., ${ }^{18}$ where the $\mathrm{Au}_{38}$ metal core is covered by 24 methylthiolate $\left(\mathrm{CH}_{3} \mathrm{~S}\right)$ molecules in such a way that the surface gold atoms form quasilinear $\mathrm{Au}-\mathrm{S}-\mathrm{Au}$ bonds, consistent with recent experimental observations. ${ }^{21}$ We consider two distinct values for the lattice parameter $a$ of the nanoparticle layer, chosen to be commensurate with either a $9 \times 9 \quad(a=22.43 \AA)$ or a $12 \times 12 \quad(a=29.97 \AA)$ graphene supercell, as shown in Figs. 1(b) and 1(c). In the first case, the nanoparticle coverage has a density of $0.230 \mathrm{~nm}^{-2}$, and in the second case, $0.129 \mathrm{~nm}^{-2}$. The geometries of the $\mathrm{Au}_{38}$ nanoparticle layers and that of the graphene layer were optimized separately, and then assembled together at a layer-to-layer distance that minimizes the total energy of the nanoparticle+graphene system. For both coverage considered, the smallest distance between a hydrogen atom of the functionalized nanoparticle and a carbon atom of the graphene layer is $2.9 \AA$ at the optimized geometry, which indicates a weak interaction between the nanoparticles and the graphene layer.

In the upper panel of Fig. 2 we show the band structure of an isolated $9 \times 9$ graphene supercell near the Fermi level, ${ }^{22}$ and in the lower panel we show the band structure of the corresponding nanoparticles + graphene system shown in Fig. 1(b). The band structure of a two-dimensional methylthiolate 

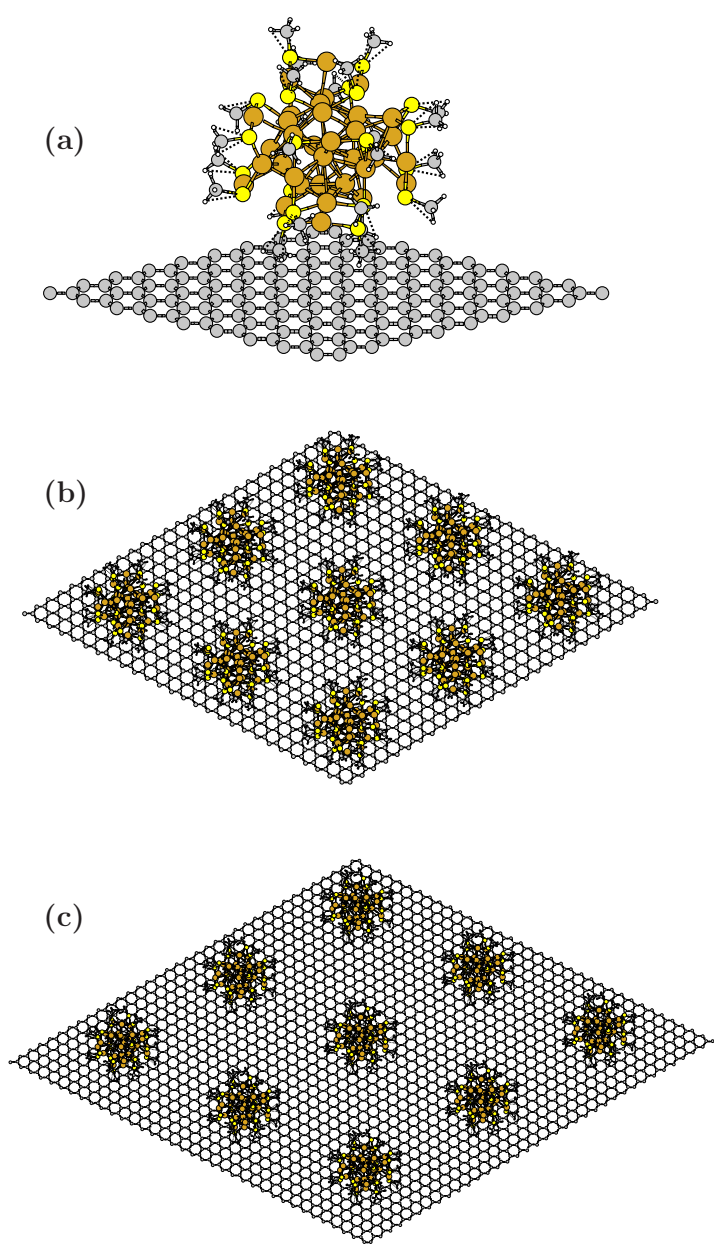

FIG. 1. (Color online) (a) Unit cell of the system composed of $\mathrm{Au}_{38}\left(\mathrm{CH}_{3} \mathrm{~S}\right)_{24}$ nanoparticles deposited on graphene, commensurate with a $9 \times 9$ graphene supercell; (b) array of those unit cells, shown for the visualization of the nanoparticle lattice; and (c) array of unit cells that are commensurate with a $12 \times 12$ graphene supercell.

functionalized $\mathrm{Au}_{38}$ film is dispersionless (as it has been previously reported in Ref. 23). Therefore, the flat bands in Fig. 2(b) can be recognized as the electronic states of the nanoparticle film. The interaction between the functionalized nanoparticles layer and the graphene layer is too small to lead to a detectable band-gap opening on the (otherwise metallic) electronic structure of the graphene layer near the $\Gamma$ point, as seen in Fig. 2. Due the differences between the electroaffinities of the functionalized nanoparticles and that of graphene, a net negative charge (of $0.3 e$ /particle) is transferred from the nanoparticles to the graphene layer. As a result, the energy of the point in $k$ space, where the upper valence band and the lowest conduction band are degenerate (the Dirac point), does not coincide with the Fermi level $E_{F}$ as in the isolated, neutral graphene (see upper panel of Fig. 2 ), but it rather shifts downward relative to $E_{F}$, as shown in the bottom panel of Fig. 2(b). Apart from the shift in $E_{F}$ due to this charge transfer, and from minor anticrossing couplings on the order of $0.01 \mathrm{eV}$, at crossing points between the (flat) nanoparticle states and the graphene states, the resulting band structure of the graphene+nanoparticles system near the $\Gamma$ point is essentially a superposition of the band

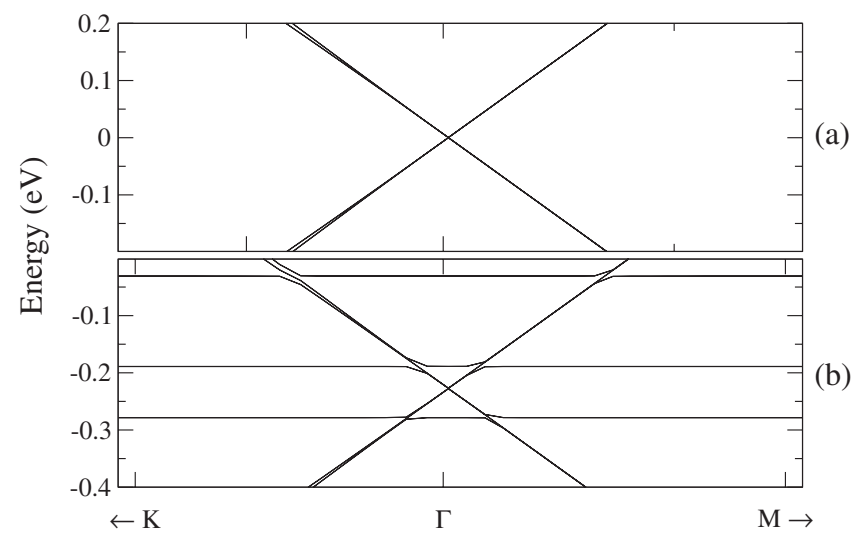

FIG. 2. (a) Band structure, near the $\Gamma$ point of a $9 \times 9$ graphene supercell. (b) Band structure, near the $\Gamma$ point of the nanoparticles + graphene system shown in Fig. 1(b). In both panels the Fermi level is set to zero and the band structures were calculated along the $\mathrm{K}-\Gamma-\mathrm{M}$ directions.

structures of the isolated systems. This is shown in Fig. 2.

In Fig. 3(a) we show the band structure of an isolated $12 \times 12$ graphene supercell near the Fermi level, and in Fig. 3 (b) we show the band structure of the nanoparticles + graphene system commensurate with the $12 \times 12$ graphene supercell [shown in Fig. 1(c)]. Similar to the case with denser coverage, considered in the previous paragraph, no band gap near the $\Gamma$ point is opened in the functionalized nanoparticles+graphene system, keeping the metallic character of the graphene. Also similar to the previous case, the band structure of the nanoparticles+graphene system near the $\Gamma$ point is nearly a superposition of the band structures of the isolated systems, and a net negative charge (of $0.35 e /$ particle) is transferred from the nanoparticles to the graphene layer, resulting in an energy shift in the Dirac point relative to $E_{F}$.

\section{B. Effects of additional charge}

We have also investigated the possible changes in the electronic structure of graphene due the inclusion of addi-

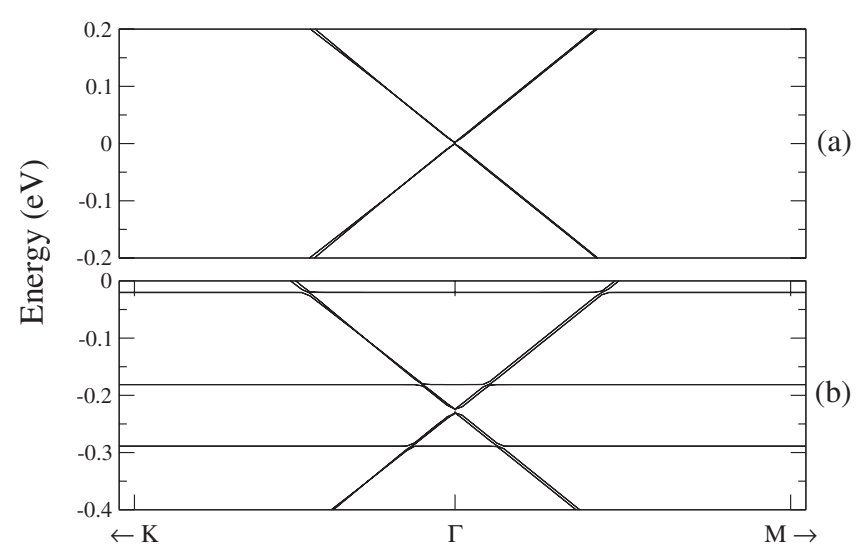

FIG. 3. (a) Band structure, near the $\Gamma$ point of an isolated 12 $\times 12$ graphene supercell. (b) Band structure, near the $\Gamma$ point of the nanoparticles + graphene system shown in Fig. 1(c). In both panels the Fermi level is set to zero and the band structures were calculated along the $\mathrm{K}-\mathrm{\Gamma}-\mathrm{M}$ directions. 


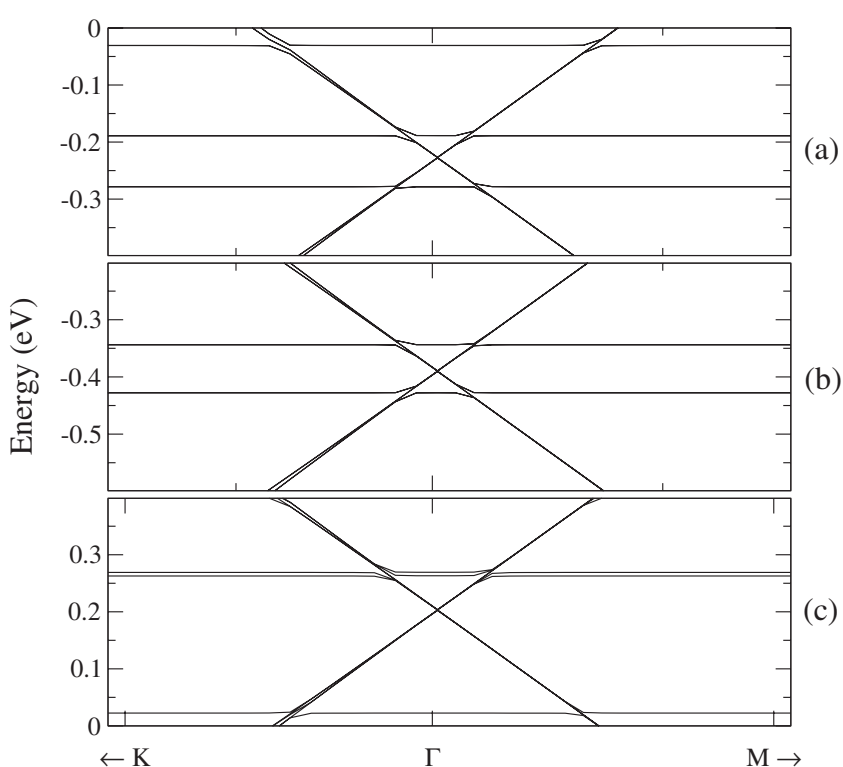

FIG. 4. Energy bands, near the $\Gamma$ point of the nanoparticles + graphene system shown in Fig. 1(b) in several charge states: (a) uncharged; (b) charged with an additional electron; and (c) charged with an additional hole. In all panels, the Fermi level is set to zero and the energy bands are calculated along the $\mathrm{K}-\mathrm{\Gamma}-\mathrm{M}$ directions.

tional charge in the graphene+nanoparticles system. Figure 4 shows the band structures of (a) the neutral $9 \times 9$ graphene + nanoparticles system, (b) the system charged with an extra electron, and (c) the system charged with an extra hole. Upon the addition of an electron [Fig. 4(b)], both the Dirac point and the dispersionless bands of the nanoparticles film are shifted to lower energies, relative to the Fermi energy, by nearly the same amount. This is associated to the fact that the additional negative charge is equally shared between the graphene layer and the nanoparticle film, as indicated by a Mulliken population analysis. The effect of an additional hole in the system is different from that of an additional electron. The extra positive charge is not equally shared between the nanoparticles film and the graphene layer: $70 \%$ of the additional positive charge is localized at the nanoparticle. As a result of such asymmetric positive charging, the relative position between the dispersionless nanoparticles bands and the graphene bands are changed due the addition of an extra hole in the system, as it is possible to see in Fig. 4(c).

As it is also possible to see in panels (b) and (c) of Fig. 4, the additional electron or hole in the system does not open a band gap on the band structure of the graphene layer near the $\Gamma$ point. This suggests that the long-range nature of the additional Coulomb potential centered at the nanoparticles is unable to break the $A-B$ sublattice symmetry that makes graphene metallic, or that the metallic screening due to the graphene layer further reduces this symmetry breaking.

\section{Effects of an applied electric field}

In an another attempt to modify the electronic structure of the nanoparticles + graphene system, we added a periodic zigzag potential along the direction perpendicular to the graphene plane, namely, $\hat{z}$ direction, which corresponds to a

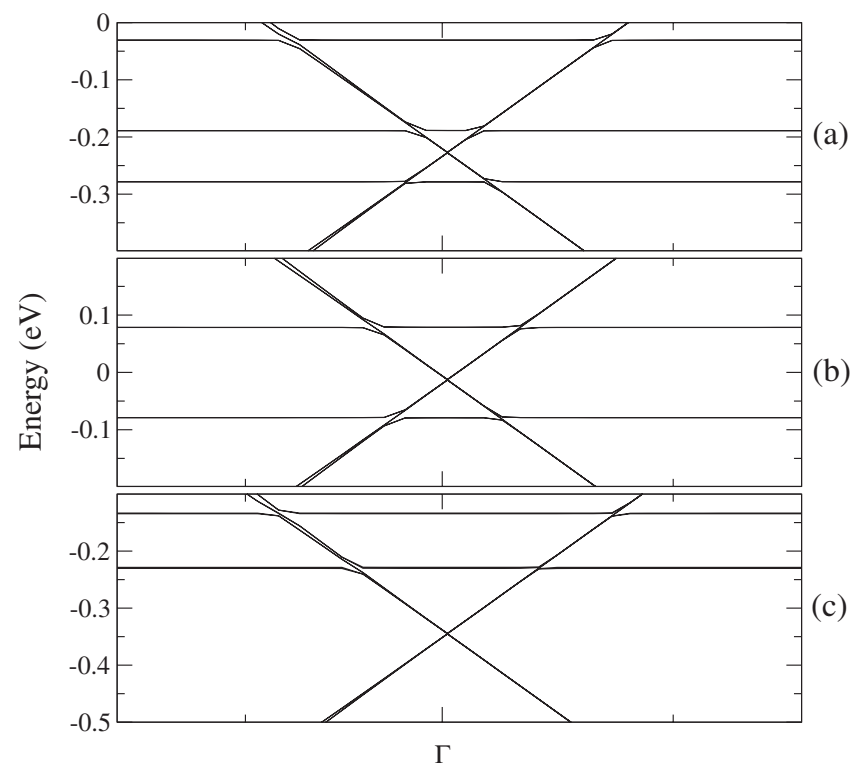

FIG. 5. Energy bands near the $\Gamma$ point of the nanoparticles + graphene system shown in Fig. 1(b) under applied electrical fields of (a) $E_{z}=0$, (b) $E_{z}=-0.5 \mathrm{~V} / \AA$, and (c) $E_{z}=0.5 \mathrm{~V} / \AA$.

constant external electric field along this direction. We considered $E_{z}$ components of the electric field ranging from -0.5 up to $0.5 \mathrm{~V} / \AA$. Figure 5 shows the band structures of the $9 \times 9$ graphene+nanoparticles system for $E_{z}=0, E_{z}$ $=-0.5 \mathrm{~V} / \AA$, and $E_{z}=0.5 \mathrm{~V} / \AA$. Depending on the sign of $E_{z}$, electrical charge is transferred either from graphene to the nanoparticles film (negative $E_{z}$ ), or vice-versa (positive $\left.E_{z}\right)$. Concerning the electronic structure, the charge transferred from one part of the system to the other leads to opposite shifts in the graphenelike bands and of the nanoparticlelike bands. However, Fig. 5 also shows that the applied field does not open a band gap in the graphenelike electronic states near the $\Gamma$ point.

\section{New Dirac points and minigaps}

Park et $a l .{ }^{24}$ have recently predicted that, when a periodic potential with triangular lattice symmetry is applied to graphene, new Dirac points are generated at the $M$ point of

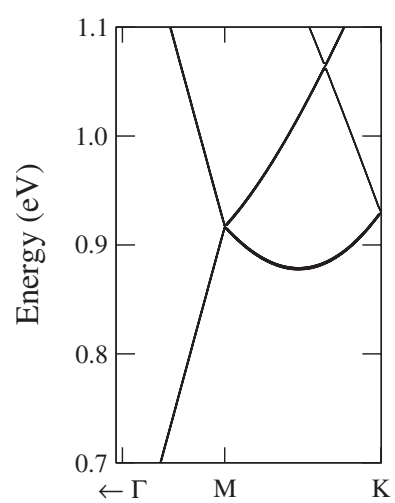

(a)

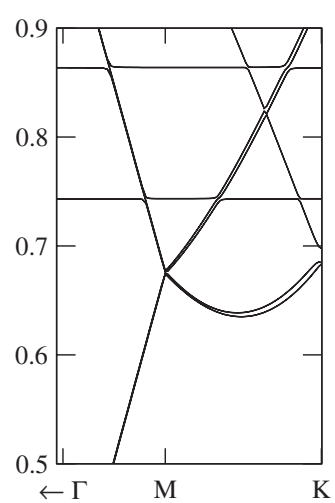

(b)
FIG. 6. Energy bands of (a) an isolated $9 \times 9$ graphene supercell and of (b) the nanoparticles+graphene system shown in Fig. 1(b), near the $M$ and $K$ points of the system supercell. 


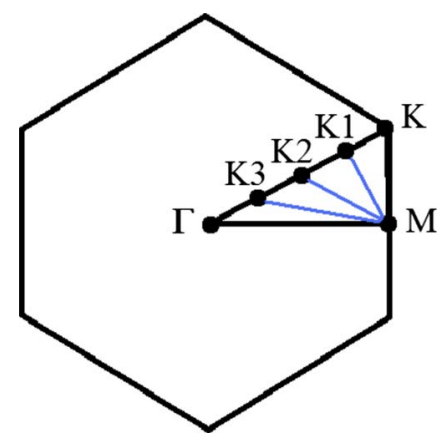

FIG. 7. (Color online) Five distinct linear paths in the first BZ that start at the $M$ point and end, respectively, at the points $K, K 1$, $K 2, K 3$, and $\Gamma$.

the system supercell, with anisotropic group velocity. For specific potentials, they also predicted that these new Dirac points can fall in an energy-gap region of the remaining electronic states. Figure $6(\mathrm{~b})$ indicates that our proposed $9 \times 9$ graphene+nanoparticles system can lead to a new Dirac point at the $M$ point, at about $0.7 \mathrm{eV}$ above the Fermi energy (and at about $0.9 \mathrm{eV}$ above the original graphene Dirac point). This results from the folding of the electronic structure of the original graphene layer into the supercell, as seen in Fig. 6(a).

The new Dirac point at the $M$ point is anisotropic, as predicted by Park et al. ${ }^{24}$ To demonstrate that, we consider five distinct linear paths in the first Brillouin zone (BZ) that start at the $M$ point and end, respectively, at the points $K, K 1$, $K 2, K 3$, and $\Gamma$, as indicated in Fig. 7. The band dispersions along those paths, shown in Fig. 8, clearly demonstrate that the feature at the $M$ point has the topology of a Dirac point, and that it is anisotropic, that is, its dispersion depends on the direction on the reciprocal space.
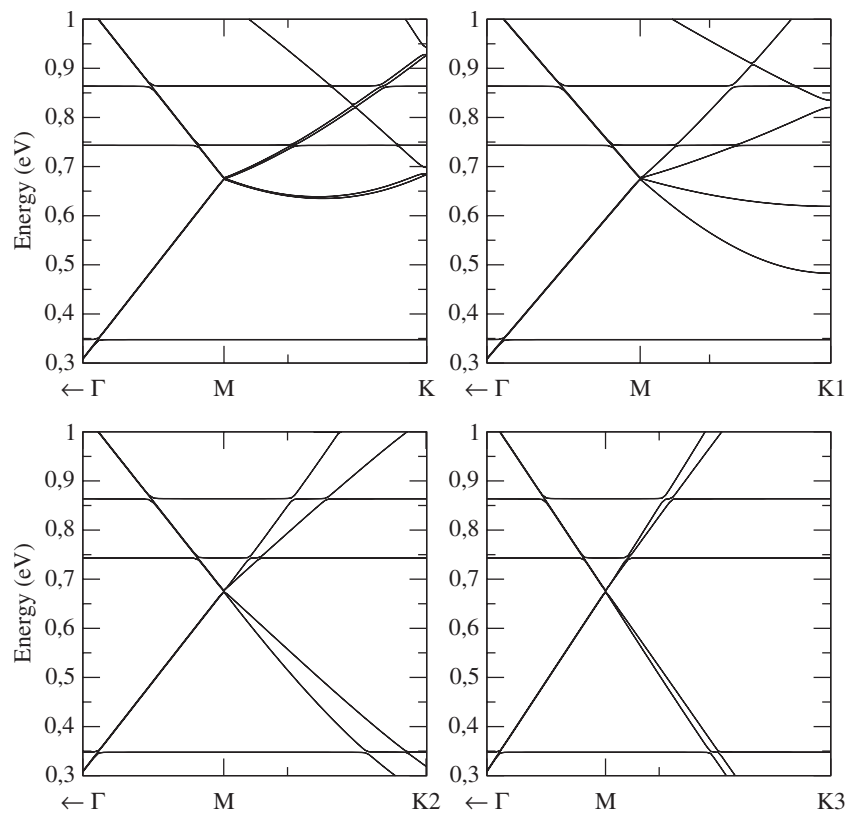

FIG. 8. Energy bands, near the $M$ point of the nanoparticles + graphene system shown in Fig. 1(b), along the paths indicated in Fig. 7.
We can also see in Fig. 6(b) that a minigap is opened at the $K$ point. The new Dirac-type point at $M$ almost falls into the minigap region. For the nanoparticles + graphene system commensurate with the graphene $12 \times 12$, we also find a similar new Dirac point at $M, 0.69 \mathrm{eV}$ above the original Dirac point. The ratio between this value and the corresponding value for the $9 \times 9$ supercell, $0.69 / 0.92=0.75$, is the same as the inverse of the ratio of the corresponding lattice parameters. This indicates a $1 / a$ scaling of the energy position of the new Dirac points, relative to the original Dirac point.

\section{E. Deposited layers of bare nanoparticles}

As described in the previous paragraphs, the graphene bands are only weakly affected at the $\Gamma$ point by the interaction with methythiolate-capped gold nanoparticles. This is partly due to the relatively large distances between the nanoparticle Au cores and the graphene carbon atoms, which leads to a slowly varying perturbing potential on the graphene layer that is unable to break the $A-B$ graphene lattice symmetry and, therefore, to open a gap on the graphene electronic structure. In an attempt to reduce the Au-carbon distance, we have investigated the interaction of graphene

(a)
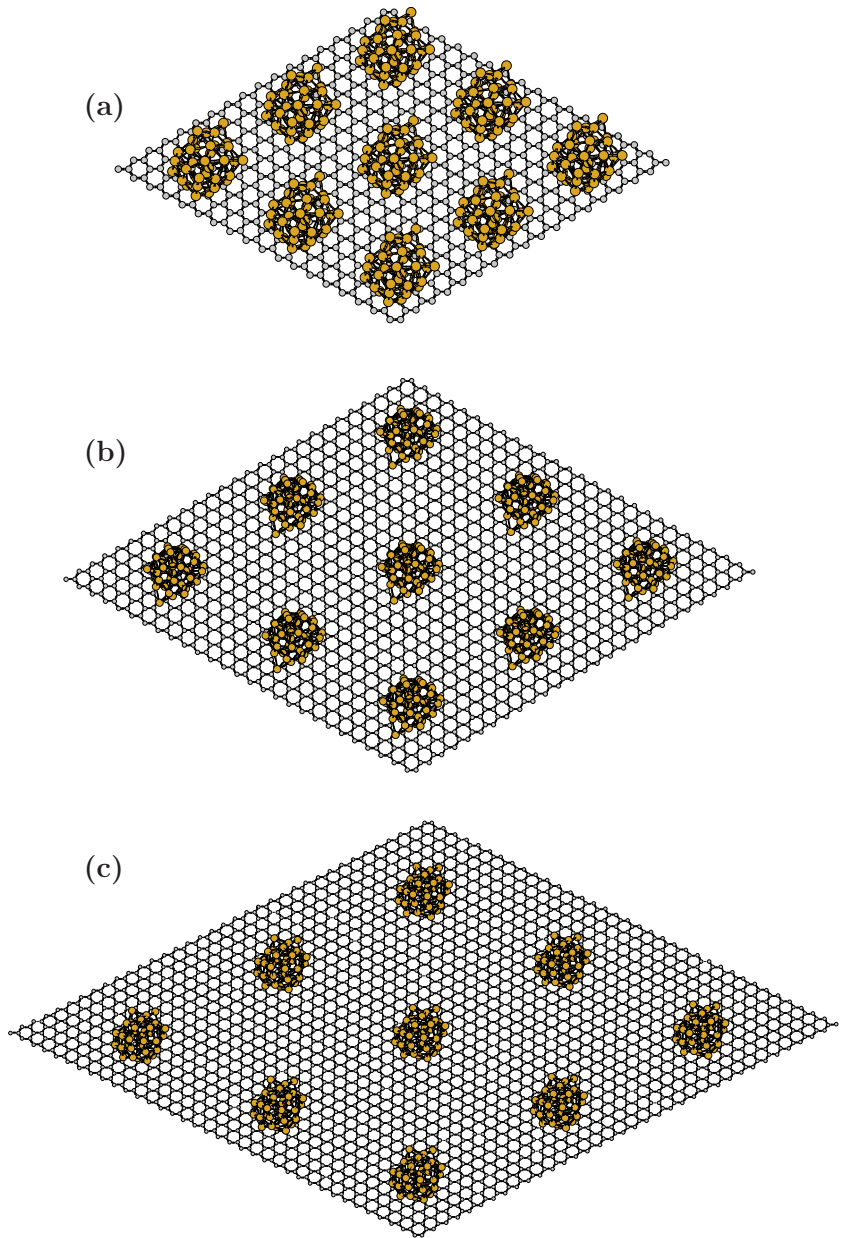

FIG. 9. (Color online) Arrays of (a) $6 \times 6$, (b) $9 \times 9$, and (c) $12 \times 12$ unit cells of graphene covered by bare $\mathrm{Au}_{38}$ nanoparticles. 


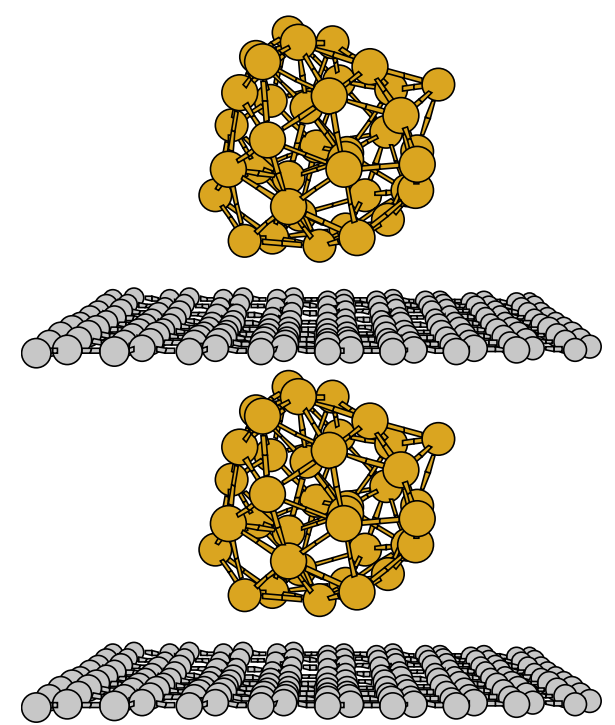

FIG. 10. (Color online) Unit cell of the system constituted by a bare $\mathrm{Au}_{38}$ nanoparticle deposited on $9 \times 9$ graphene and one of its periodic images along the $z$ direction.

with bare (noncovered) $\mathrm{Au}_{38}$ nanoparticles. In contrast with thiolate-capped $\mathrm{Au}$ nanoparticles, which spontaneously assemble into periodic two-dimensional triangular lattices, ${ }^{11}$ we do not expect that bare Au nanoparticles on graphene would spontaneously form periodic lattices: a chemical route involving thiol desorption, for instance, might achieve that goal. We note that there are already experimental reports of chemical desorption of thiols from Au nanoparticle films on graphite, leaving thiol-free nanoparticles that do not collapse by sintering. ${ }^{25}$ Physical deposition routes are also possible. For instance, the physical deposition of small coverage of $\mathrm{Au}$ over graphite by molecular-beam epitaxy ${ }^{26}$ leads to isolated Au clusters, most within a narrow distribution of diameters between 0.5 and $0.7 \mathrm{~nm}$. In our calculations, we first consider an amorphous $\mathrm{Au}_{38}$ cluster (which is energetically more stable than its ordered octahedral isomer $)^{18}$ that is periodically repeated in a triangular lattice that is commensurate with an underlying $9 \times 9$ graphene supercell [see Fig. 9(b)]. The geometry of this system was fully optimized until the remaining forces were smaller than $0.05 \mathrm{eV} / \AA$. The optimized unit cell and one of its periodic images along the $\hat{z}$ direction are shown in Fig. 10. As the figure shows, the geometry optimization leads to a strong reduction in the c/a ratio of the supercell, which now represents a periodic $\mathrm{Au} /$ graphene heterostructure along the $\hat{z}$ direction. The minimal distance between a gold and a carbon atom is $\approx 2 \AA$, which indicates a strong interaction between the bare Au nanoparticles and graphene, as compared to the weak interaction in the case of the functionalized nanoparticles.

The strong, short-range interaction between the bare gold clusters and the $9 \times 9$ graphene supercell leads to a band-gap opening of $24 \mathrm{meV}$ near the $\Gamma$ point, between the graphenelike bands that previously touched at the Dirac point, as seen in Fig. 11(a). The energy-gap region is below the Fermi energy due to electron transfer from the nanoparticles to graphene and, therefore, the neutral system is still metallic. Upon a proper $p$-doping, however, the Fermi energy can be

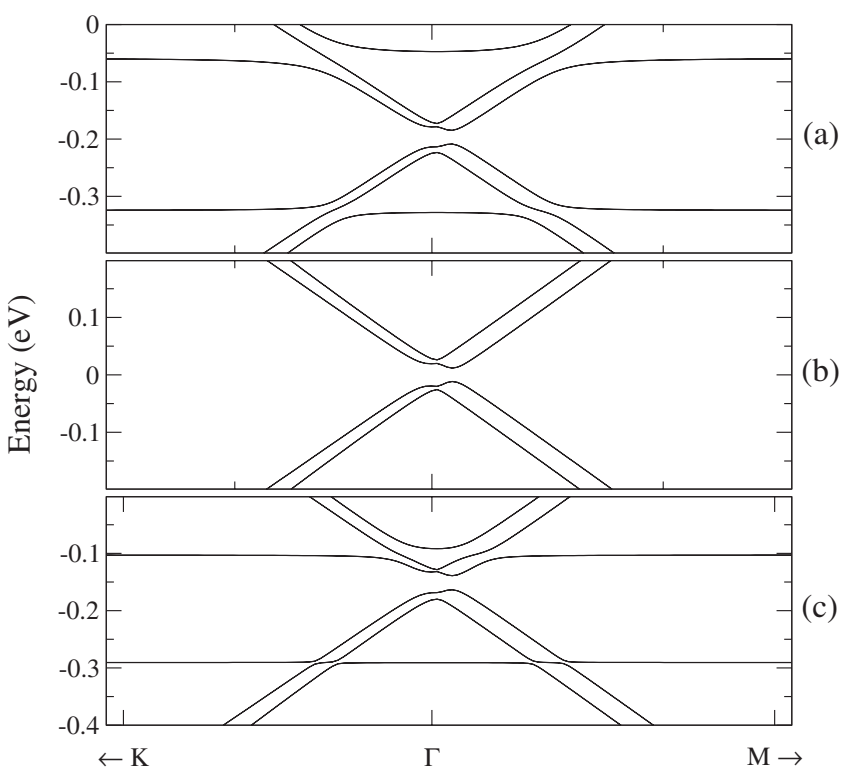

FIG. 11. Energy bands of (a) bare $\mathrm{Au}_{38}$ nanoparticles confined between successive graphene layers; (b) the isolated $9 \times 9$ graphene layer with the same (distorted) geometry as in the previous case; and (c) bare nanoparticles interacting with a single distorted graphene layer.

placed in the gap region and the graphene+ nanoparticles system would become semiconductor.

To investigate the physical origin of the band gap shown in Fig. 11(a), we calculated the band structure of two additional systems. First, we increased the $c$-axis distance of the supercell such that there are not interactions between periodic images along $\hat{z}$ direction, that is, the system is effectively a single-layer graphene plus nanoparticles. The band structure of such system, shown in Fig. 11(c), depicts the same band gap as before. Therefore, the origin of the gap is not the interaction between the graphene layers mediated by the nanoparticles. Next, we considered the band structure of a single, isolated graphene layer with the same (slightly) distorted geometry as in the graphene+nanoparticles system.

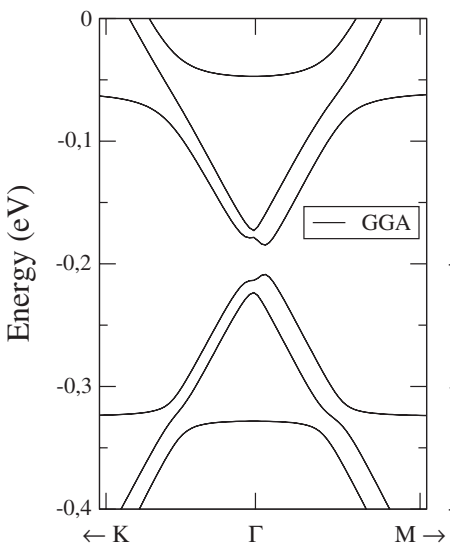

(a)

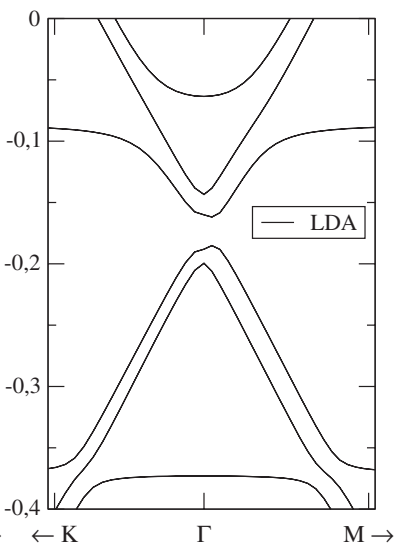

(b)
FIG. 12. Energy bands of bare $\mathrm{Au}_{38}$ nanoparticles confined between successive graphene layers obtained within either the (a) GGA or the (b) LDA approximation. 


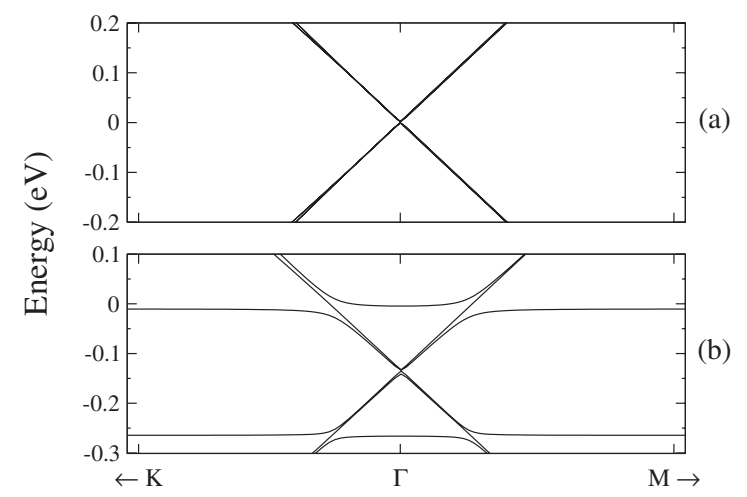

FIG. 13. Energy bands, near the $\Gamma$ point of (a) an isolated 12 $\times 12$ graphene supercell; (b) the system composed of bare, amorphous $\mathrm{Au}_{38}$ clusters and graphene shown in Fig. 9(c).

The band structure of the distorted graphene, shown in Fig. 11(b), depicts the same band gap as before. Therefore, the physical origin of the band gap is the breaking of the $A-B$ sublattice symmetry due to the localized distortions in graphene caused by the bare nanoparticles.

The results of Fig. 11, indicating the band-gap opening of a specific graphene+nanoparticles system, were performed with the use of a GGA exchange-correlation functional, as described previously. In order to verify if the band-gap opening is related to the use of a specific functional, we performed the same calculations within the local-density approximation (LDA) (Ref. 27) for the exchange-correlation functional. Figure 12 shows the band structure, near the $\Gamma$ point, of the bare nanoparticles lattice interacting with a 9 $\times 9$ graphene supercell obtained within both the GGA and the LDA approximations. Although there are some quantitative differences in the band dispersions between the two functional, we obtain the same value of the band gap, of about $24 \mathrm{meV}$, in both cases.

In order to investigate if the band-gap-opening effect is sensitive to the density of the nanoparticle coverage, we also performed calculations with both larger and smaller graphene supercells. Specifically, we considered lattices of bare, amorphous $\mathrm{Au}_{38}$ clusters commensurate with $12 \times 12$ and $6 \times 6$ graphene supercells, shown in Figs. 9(c) and 9(a), respectively. The geometry of those systems were fully optimized until the remaining forces were smaller than $0.05 \mathrm{eV} / \AA$. The minimal distance between a gold and a carbon atom is about $2 \AA$ in the $\hat{z}$ direction, similar to the 9 $\times 9$ system described in the previous paragraphs. Figure 13(b) shows energy bands near the $\Gamma$ point of the $12 \times 12$ graphene supercell interacting with the commensurate lattice of bare gold clusters, and (a) shows the corresponding band structure for the isolated graphene system. Unlike the previous result for bare nanoparticles on a $9 \times 9$ graphene supercell, in the $12 \times 12$ system no band gap is opened at $\Gamma$ due to the nanoparticles, in spite of the fact that there is a deformation in the graphene region below the nanoparticles similar to

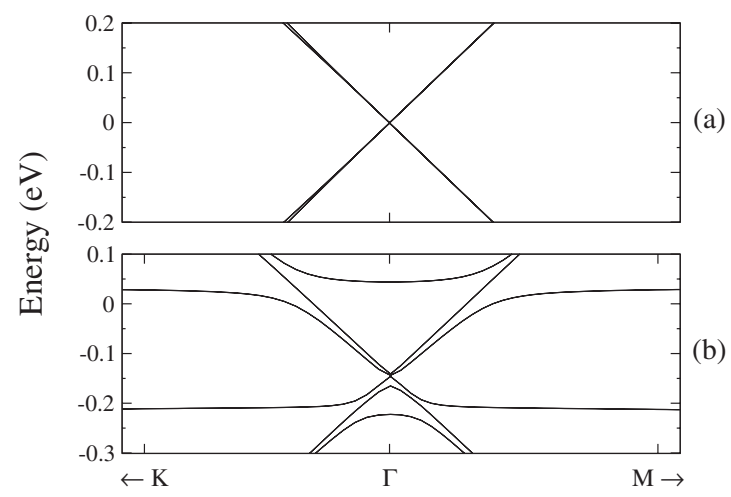

FIG. 14. Energy bands, near the $\Gamma$ point of (a) an isolated 6 $\times 6$ graphene supercell; (b) the system composed of bare, amorphous $\mathrm{Au}_{38}$ clusters and graphene shown in Fig. 9(a).

the $9 \times 9$ system. Considering that in the isolated "diluted" limit (nanoparticles very far apart from each other) no band gap can be opened due to the semimetallic nature of the graphene host system, a possible explanation for the absence of a band gap in the $12 \times 12$ system is that such limit has been reached.

The case of the lattice of bare, amorphous $\mathrm{Au}_{38}$ clusters commensurate with the $6 \times 6$ graphene supercells, shown in Fig. 9(a), is structurally distinct from the $9 \times 9$ and the 12 $\times 12$ systems: in this case, the deformation of the graphene layer induced by the nanoparticles is negligible. Consistent with that, the band structure of this system, shown in Fig. 14(b), shows that no band gap is opened near the $\Gamma$ point. Therefore, our results suggest that the nanoparticle-induced band-gap opening would only occur for an "intermediate" coverage of nanoparticles, that is, one that leads both to the deformation of the graphene layer and to a significant area of the deformed graphene relative to the undeformed, bulk graphene area between the nanoparticles.

\section{SUMMARY}

In summary, we performed a first-principles study of electronic and structural properties of two-dimensional arrays of $\mathrm{Au}_{38}$ nanoparticles, functionalized or not by methylthiolate molecules, in contact with a graphene layer. We find that the interaction of functionalized nanoparticles with the graphene layer causes electronic structure modifications in graphene such as minigaps, charge transfer, and new Dirac points. Nevertheless, this interaction does not lead to the opening of a band gap at the Dirac point, even in the case of charged nanoparticles or under applied electric field. We also find that the interaction of bare nanoparticles with graphene may cause a small band-gap opening at the Dirac point, for a moderate $\left(\approx 0.2 \mathrm{~nm}^{-2}\right)$ coverage of nanoparticles.

\section{ACKNOWLEDGMENTS}

We acknowledge the support from the Brazilian agencies CNPq, CAPES, and FAPEMIG. 
*chacham@fisica.ufmg.br

${ }^{1}$ K. S. Novoselov, A. K. Geim, S. V. Morozov, D. Jiang, Y. Zhang, S. V. Dubonos, I. V. Grigorieva, and A. A. Firsov, Science 306, 666 (2004).

${ }^{2}$ K. S. Novoselov, A. K. Geim, S. V. Morozov, D. Jiang, M. I. Katsnelson, I. V. Grigorieva, S. V. Dubonos, and A. A. Firsov, Nature (London) 438, 197 (2005).

${ }^{3}$ Y. Zhang, Y.-W. Tan, H. L. Stormer, and P. Kim, Nature (London) 438, 201 (2005).

${ }^{4}$ K. S. Novoselov, E. McCann, S. V. Morozov, V. I. Fal'ko, M. I. Katsnelson, U. Zeitler, D. Jiang, F. Schedin, and A. K. Geim, Nat. Phys. 2, 177 (2006).

${ }^{5}$ X. Li, X. Wang, L. Zhang, S. Lee, and H. Dai, Science 319, 1229 (2008).

${ }^{6}$ S. Y. Zhou, G.-H. Gweon, A. V. Fedorov, P. N. First, W. A. de Heer, D.-H. Lee1, F. Guinea, A. H. Castro Neto, and A. Lanzara, Nature Mater. 6, 770 (2007).

${ }^{7}$ S. Kim, J. Ihm, H. J. Choi, and Y. W. Son, Phys. Rev. Lett. 100, 176802 (2008).

${ }^{8}$ J. B. Oostinga, H. B. Heersche, X. Liu, A. F. Morpurgo, and L. M. K. Vandersypen, Nature Mater. 7, 151 (2008).

${ }^{9}$ T. G. Pedersen, C. Flindt, J. Pedersen, N. A. Mortensen, A. P. Jauho, and K. Pedersen, Phys. Rev. Lett. 100, 136804 (2008).

${ }^{10}$ G. Giovannetti, P. A. Khomyakov, G. Brocks, P. J. Kelly, and J. van den Brink, Phys. Rev. B 76, 073103 (2007).

${ }^{11}$ A. Ulman, Chem. Rev. (Washington, D.C.) 96, 1533 (1996).

${ }^{12}$ R. Muszynski, B. Seger, and P. V. Kamat, J. Phys. Chem. C 112, 5263 (2008).

${ }^{13}$ W. Kohn and L. J. Sham, Phys. Rev. 140, A1133 (1965).

${ }^{14}$ J. M. Soler, E. Artacho, J. D. Gale, A. García, J. Junquera, P.

Ordejón, and D. Sánchez-Portal, J. Phys.: Condens. Matter 14, 2745 (2002).

${ }^{15}$ J. P. Perdew, K. Burke, and M. Ernzerhof, Phys. Rev. Lett. 77, 3865 (1996).

${ }^{16}$ N. Troullier and J. L. Martins, Phys. Rev. B 43, 1993 (1991).

${ }^{17}$ J. Junquera, O. Paz, D. Sanchez-Portal, and E. Artacho, Phys. Rev. B 64, 235111 (2001).

${ }^{18}$ I. L. Garzón, C. Rovira, K. Michaelian, M. R. Beltran, P. Ordejon, J. Junquera, D. Sanchez-Portal, E. Artacho, and J. M. Soler, Phys. Rev. Lett. 85, 5250 (2000).

${ }^{19}$ R. J. C. Batista, M. S. C. Mazzoni, L. O. Ladeira, and H. Chacham, Phys. Rev. B 72, 085447 (2005).

${ }^{20}$ J. Moreno and J. M. Soler, Phys. Rev. B 45, 13891 (1992).

${ }^{21}$ P. D. Jadzinsky, G. Calero, C. J. Ackerson, D. A. Bushnell, and R. D. Kornberg, Science 318, 430 (2007).

${ }^{22}$ The Dirac point is at the $\Gamma$ point of the $9 \times 9$ supercell due to band folding.

${ }^{23}$ R. J. C. Batista, M. S. C. Mazzoni, I. L. Garzón, M. R. Beltrán, and H. Chacham, Phys. Rev. Lett. 96, 116802 (2006).

${ }^{24}$ C. H. Park, L. Yang, Y. W. Son, M. L. Cohen, and S. G. Louie, Phys. Rev. Lett. 101, 126804 (2008).

${ }^{25}$ D. Grumelli, C. Vericat, G. Benitez, M. E. Vela, R. C. Salvarezza, L. J. Giovanetti, J. M. Ramallo-López, F. G. Requejo, A. F. Craievich, and Y. S. Shon, J. Phys. Chem. C 111, 7179 (2007).

${ }^{26}$ Y. Kitsudo, A. Iwamotoa, H. Matsumotoa, K. Mitsuharaa, T. Nishimuraa, M. Takizawab, T. Akitac, Y. Maedac, and Y. Kido, Surf. Sci. 603, 2108 (2009).

${ }^{27}$ D. M. Ceperley and B. J. Alder, Phys. Rev. Lett. 45, 566 (1980); J. P. Perdew and A. Zunger, Phys. Rev. B 23, 5048 (1981). 